\title{
Missed Diagnosis and the Development of Acute and Late Lyme Disease in Dark Skinned Populations of Appalachia
}

\author{
James R Palmieri*, Anushri Kushwaha-Wagner, Abe-Melek Bekele, Jasyn Chang, Alison \\ Nguyen, Nathanael N Hoskins, Raakhi Menon, Mohamed Mohamed and Susan L Meacham \\ Department of Osteopathic Medicine, USA
}

*Corresponding author: James R Palmieri, Department of Biomedical Sciences, Edward Via College of Osteopathic Medicine,

Virginia Campus. USA

\section{ARTICLE INFO}

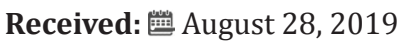

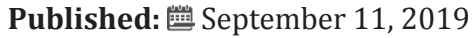

Citation: James R Palmieri, Anushri Kushwaha-Wagner, Abe-Melek Bekele, Jasyn Chang, Alison Nguyen, et al. Missed Diagnosis and the Development of Acute and Late Lyme Disease in Dark Skinned Populations of Appalachia. Biomed J Sci \& Tech Res 21(2)-2019. BJSTR. MS.ID.003583.

Keywords: Lyme Disease; Dark Skinned; Missed Diagnosis; Appalachia; Minority; Late Lyme Disease; Chronic Lyme Disease
ABSTRACT

Background: Lyme Disease (LD) is the most commonly reported vector-borne disease in the United States, affecting over 300,000 people in the United States each year. If early LD goes undetected or is inadequately treated, the causative spirochete bacteria, Borrelia burgdorferi, can disseminate throughout the body and cause chronic symptoms that will characterize a patient with late LD. The incidence of LD is generally reported at a higher rate in light-skinned patients as compared to dark-skinned patients.

Aim: To assess the rate and causative factors of late Lyme Disease in dark-skinned individuals within the Appalachian region and encourage research into the need for early clinical evaluation and testing for at-risk patients.

Discussion: Healthcare providers are at risk of missing the diagnosis of acute Borrelia infection in dark-skinned patients based on historically lower reporting frequency and non-traditional early symptoms and clinical presentation. Misdiagnosis, or delayed diagnosis, adversely affects the health outcomes of people of color and risks progression of the infection from early to late LD.

Recommendations: We defined the characteristics of darker skin tones as a quantitative score to identify the risk for non-traditional presentation of the early signs of Borrelia infection. Using this score, we recommend determining the association between dark skin color and undiagnosed LD based upon a systematic study on the correlation of patient skin color and positive LD testing. This data should be combined with other risks of exposure. The results of the recommended research would determine whether physicians in Appalachia should incorporate comprehensive treatment plans for LD in dark-skinned patients as preventive medical practice.

\section{Lyme Disease Defined}

According to the Centers for Disease Control and Prevention (CDC) presently, Lyme Disease (LD) is the most commonly reported vector-borne disease in the United States, affecting over 300,000 people in the United States each year [1]. LD is not only of nationwide concern, but also infects people in more than 80 countries worldwide [2]. LD is commonly transmitted by the blacklegged tick, Ixodes scapularis, infected with the spirochete bacteria, Borrelia burgdorferi [1]. However, LD spirochetes are also transmitted globally through minor Borrelia species such as $B$. garinii, B. afzelii, B. valaisiana, B. lusitaniae, B. japonica, B. tanukii, B. turdi, B. miyamotoi and B. mayonii $[3,4]$.

According to the CDC in 2016, the highest incidence of reported cases of B. burgdorferi transmitted LD in the U.S. occurs in the New England region and Midwestern regions such as Wisconsin and Minnesota [1,5]. B. mayonii was recently reported in 2013 and is a genetic strain that is a mixture of $B$. burgdorferi, B. garinii, and $B$. afzelii [6]. Currently, B. mayonii resides in the Midwestern region of the United States and is the only other known Borrelia species to cause LD in the United States [6]. 
Outside of the United States, LD occurs mostly in Europe and Asia. In Europe, the majority of the LD reports appear from Scandinavian countries and Central Europe [4]. In Europe, LD cases are primarily caused by B. afzelii, followed by B. garinii, B. valaisiana and B. lusitaniae [3,4-5]. In Asia, specifically Japan, B. japonica, $B$. tanukii, B. turdi, and B. miyamotoi are the causative agents of LD $[3,4]$. While multiple Borrelia species contribute to LD globally, $B$. burgdorferi remains the primary causative agent of LD in the United States [1].

Patients infected with LD can present with a variety of symptoms [7]. During the early phase of LD, 50\% of patients get a non-itchy rash, but only $9 \%$ of people get the characteristic bullseye, Erythema Migrans (EM), usually centered on the tick bite site. Other symptoms that manifest during the acute phase of LD are flu-like symptoms that range from fevers, chills, sweats, muscle aches, fatigue, nausea, and joint pain [8]. A more severe symptom, especially in younger patients, involves peripheral cranial nerve VII, causing facial drooping, a condition resulting in unilateral or bilateral facial palsy, commonly known as Bell's Palsy [8].

If $\mathrm{LD}$ goes undetected or is improperly treated, the spirochete bacteria can disseminate throughout the body and various chronic symptoms may occur that will characterize a patient with chronic, or late phase, LD [8]. Late LD symptoms include but are not limited to: malar flush, red earlobes, temporomandibular joint dysfunction, jaw pain, severe headaches, neck stiffness, additional EM rashes on other areas of the body, arthritis, severe joint pain, facial palsy, intermittent pain in tendons, muscles, joints, and bones, heart palpitations, dizziness, shortness of breath, encephalomyelitis, meningitis, neuritis, shooting pains, numbness, or tingling in the hands or feet, depression and problems with short-term memory [7-9]. It should be noted that as of the last decade, "chronic Lyme disease" has been considered an inadequately descriptive term which would be more accurately described as "late Lyme disease" [10]. The two phrases are commonly interchanged, and, in this paper, they are used synonymously to maintain accurate reporting of survey data.

\section{Vector of Lyme Disease in Appalachia}

In the U.S. Appalachian region, the primary bacterial species that transmits LD is B. burgdorferi $[12,13]$. The vector for LD in the Appalachian region is the Ixodes scapularis tick species and is typically found in regions with mesic forests and alfisol type soils, a soil that is leached basic or slightly acid with a clay-enriched B horizon subsoil, overlying sedimentary rocks [13]. These conditions are characteristic of mountainous towns and communities found in the Appalachian region especially above 3,000 feet. Besides LD, $I$. scapularis serves as a vector for anaplasmosis, babesiosis, Borrelia miyamotoi disease, Powassan virus disease, and ehrlichiosis associated with Ehrlichia muris eauclarensis [14].

Approximately $80 \%$ of LD cases occur in endemic regions, specifically the northeast United States [15]. The majority of the remaining cases occur in the southeast and Appalachian regions. Between 2000 and 2014, the geographic distribution of LD cases significantly expanded in the southeastern United States, especially in the southern Virginia mountain ranges [11,15-17]. This spread is expected to continue further south in Appalachia over upcoming years. The cause of this continued increase of LD incidence is unknown at the time, but some hypotheses include climate change and increases in population in endemic regions [11]. Tick abundance is correlated with higher altitudes because of climate change [18]. However, one study anticipates a $213 \%$ increase in suitable habitat regions for I. scapularis because of climate change [19]. This study also reports that the southern states of the U.S. will become non-habitable for I. scapularis due to temperature increase, and therefore expects the prevalence of LD to increase in northern regions of North America [19].

\section{Appalachia and its Ethnic History}

Appalachia is a 205,000 square mile region that surrounds the Appalachian Mountains sections from southern New York to northeastern Mississippi. Geographically, this region includes all of West Virginia and parts of 12 other states: Alabama, Georgia, Kentucky, Maryland, Mississippi, New York, North Carolina, Ohio, Pennsylvania, South Carolina, Tennessee, and Virginia [20]. Ethnically, the majority of the region's earliest settlers came from Anglo-Scottish border countries (Scottish-Irish) with a minority consisting of Swedish and Finnish settlers [21]. In the 19th century, major pioneer groups included Germans along with Italians and Eastern Europeans. African Americans have been a consistent minority representative, consisting of $8 \%$ of the Appalachian Research Council (ARC)-designated region, and Native Americans, Cherokee Indians in particular [22,23].

A racial group of interest with respect to the incidence of LD in Appalachia are the Melungeons. Melungeons are an isolated tri-racial group of mixed African, European, and Native American ancestry associated with the Cumberland Gap area of central Appalachia, including portions of East Tennessee, Southwest Virginia, and eastern Kentucky [24,25]. The origins of the Melungeons came from the principle of "partus sequitur ventrem" which became Virginia Law in 1662 that deemed children to be classified by the ethnicity of the mother. This led to the union between black men and white women, which became the foundation for the oldest free families of color [26]. According to genealogists, the earliest lineage came from the unions of black and white indentured servants prior the widespread rise of slavery and with the later passing of laws that restricted interracial marriages, the children of these groups intermarried with each other or mixed with persons of Native American ancestry [25-27]. This diversification of the population led to a variety of physical traits, including skin tone, within the Appalachian region. This is of medical significance due to the historical challenge of diagnosing dermatological presentations of diseases such as LD. 


\section{The Appalachian Trail: Exposure to LD Vectors}

Cases of LD in Virginia have increased in frequency since 2005 [20]. Unique to the northeastern states with the highest prevalence of LD is the 2,175-mile Appalachian Trail (AT). Its route transects the eastern United States, passing through Virginia to Springer Mountain in Georgia, and is an ideal geographic area for LD acquisition because of the presence of the tick vector and rodent and deer hosts [21-23]. Many parents with infants and young children use the AT for day trips or short weekend camping. Epidemiological data of LD reported for counties along the AT shows that the highest rates of LD occur in New York, Connecticut, and New Jersey, and incidence rates in these states have increased since 1992 [2426]. With millions of visitors, day hikers, and section hikers that frequent various sections of the trail, interaction between hiker and tick, and thus exposure to the vectors of LD, is likely [27].

According to the study done by Knoll et al. [26], 23 out of 379 hikers reported that a rash developed during the hike. They concluded that the LD identification abilities of hikers remain poor with only $54 \%$ able to identify the surrogate marker of EM bullseye [28]. Regardless of geographic region, previous studies report a risk of exposure to spirochete-infected host-seeking I. scapularis nymphs in recreational settings including the Appalachian Trail [29]. However, the epidemiological surveillance conducted at the county spatial scale by Eisen et al. [28] demonstrated that despite the elevated risk of tick contact in national parks, one cannot adequately infer the risk of tick-borne disease for park visitors and hikers $[29,30]$. Due to varied ecology and varied human behaviors within parks, the density of infected ticks may further obfuscate any real correlations and findings [31].

\section{Racial Differences in Reported LD Incidence}

Skin color is classified utilizing either the Fitzpatrick scale or the older von Luschan scale. The Fitzpatrick scale consists of six roman numerals that categorize epidermal melanin based on its response to the effects of sun and Ultraviolet (UV) light, whereas the older von Luschan scale visually evaluates a subject's skin color by comparing it to a standardized set of thirty-six opaque tiles [32]. The von Luschan scale would be a more relevant tool for the study of direct skin color, while the Fitzpatrick scale focuses on the response of variously pigmented skin to UV exposure [33]. Drawing a parallel between skin color and specific ethnic groups is difficult, controversial, and often unreliable.

In most states including Virginia, there are five categories to indicate one's racial designation and this information can be readily acquired [34]. However, this information is often inadequate due to ethnic groups having a substantial variation in skin color. For example, Virginia has an Asian population of $6.1 \%$, but they could fall under any of the Roman numerals in the Fitzpatrick scale [35]. As reported by Fix et al [34] from 1993-2000, the incidence of LD was inordinately higher among the white population [36]. In this investigation, the data for race was compiled via self-designation, but this method is prone to errors as the classification process is not well defined. However, using this method does allow LD to be analyzed in conjunction with skin color rather than race alone [36,37].

Since skin color and ethnicity is difficult to accurately compare and contrast with the diagnosis of LD and with the presence of erythema migrans, the Maryland State LD Registry was established in 1993 by the University of Maryland, Baltimore, and the Department of Health and Mental Hygiene (DHMH). Their goal was to provide a database for assessing outcomes of antibiotic therapy and to provide thorough, statewide surveillance for LD during 1993-1996. The registry was heavily patient driven and was inclusive to the different kinds of people who were affected by LD [36-39]. Presently, Lymedisease.org, an educational and research advocacy group, has established MyLyme Data to develop and to accelerate research in LD by providing observational data and by serving as a research platform for more traditional LD studies. Most of the patients in MyLyme Data (79\%) identify their current stage of illness as late (16\% of patients) or as chronic LD (61\% of patients). This registry is designed to address all sorts of factors such as disease progression, delayed diagnoses, factors contributing to poor outcomes, geographical prevalence, ethnicity and skin color, treatments, genetic factors, and studying those who made a full recovery [40].

\section{Reporting of LD Incidence for Individuals with Dark Skin}

The incidence of LD is generally reported at a higher rate in white people as compared to other races [36-41]. This high incidence of LD is attributed to living in an endemic area, which is regarded as a key risk factor due to increased risk of exposure [36]. Although people with lighter skin and people of color both live in endemic areas, this disparity in the reporting of LD incidence persists [42]. According to a study on the racial differences in reported LD incidence, this disparity could be due to a number of factors including risk exposure, disparities in access to healthcare, misdiagnosis, and health provider bias [36]. The characteristic EM rash does not always appear with LD, but if present, the rash can be difficult to see on darker skin $[43,44]$. The possible result of misdiagnosis or the inability to appreciate EM could explain the increased occurrence of arthritis among African Americans in an endemic area as arthritis is an indication of the late disseminated stage of LD [36-46]. The study on racial differences in reported LD incidence also found a negative association between the LD symptoms of EM and arthritis [36]. As LD spreads towards the southwestern part of Virginia, health providers, as well as the general population, need to have an increased awareness of how LD manifests in people of color so that infected individuals may receive proper treatment [47].

\section{Differences in Exposure Risks for Individuals with Dark Skin}

The disparity of LD incidence between people with light skin and people with darker skin has been correlated to LD exposure 
risk [36]. A study conducted from 2000-2014 to characterize LD expansion in Virginia found that the most notable area of LD expansion was along the Appalachian Mountains [11]. They found that this trend increases exposure risk for individuals living along this mountain chain to certain tick habitats ranging from suburban to rural locations. According to a 2011 study conducted by the National Park Service, 92\% of visitors who went to Shenandoah National Park were white [48]. In regard to risk exposure, a 2018 Outdoor Participation Report by The Outdoor Foundation found that $74 \%$ of people who engaged in at least one of forty-two different outdoor activities in 2017 were non-Hispanic Caucasian, while 10\% were Hispanic, 9\% were Black, and 6\% were Asian [49]. Racial differences in outdoor activities appears to be a significant factor in the racial disparity in LD incidence rates [36-49]. However, underrecognition of $L D$ in people with dark skin seems to also be a likely significant factor in LD incidence disparity as LD is underreported by as much as $90 \%$ and is more difficult to diagnose than in people with light skin [11-36].

When statewide surveillance of LD began in Virginia in 1989, the practice of confirming LD cases was with the presence of EM or the correlation of laboratory testing with at least one late stage symptom. This practice continued until 2008, when the National Surveillance Case Definition changed [50]. The current practice to confirm a LD case in an already endemic area is with the presence of EM or known exposure; otherwise, a positive laboratory testing is required in a non-endemic area for the confirmation of $\operatorname{LD}[50,51]$. Despite the change, these guidelines still could lead to an underdiagnosis of dark-skinned individuals with LD due to the differing appearance of the classic EM.

\section{Manifestation of LD Differences in Ethnic Groups}

LD is known as the "second great imitator" after systemic erythematous lupus, due to its numerous nonspecific symptoms [51]. The EM rash is the only physical symptom that is distinctive enough to allow a diagnosis of LD in the absence of laboratory testing [49]. It is important to note that on darker skin, EM resembles a bruise [52]. Currently, laboratory tests alone for LD are not reliable and require confirmation by a clinical diagnosis [43]. As LD persists untreated, it causes a late disseminated infection with symptoms including arthritis, arrhythmia, encephalopathy, carditis, and severe headaches which are more prevalently observed in individuals with darker skin since the EM rash is difficult to observe [45-53]. Hispanics with LD, for instance, were significantly more likely than non-Hispanics with LD to have signs of disseminated infection and symptom onset matching the criteria for late phase or chronic LD [54]. This could suggest an association between the presence of an LD infection and the related symptoms. Although diagnostic tests should be performed and appropriate therapy could be administered to patients with symptoms of LD, the difficulty of diagnosing LD lies in health providers' awareness of how LD manifests in populations of darker skin color [46].

\section{The Role of Healthcare Providers in LD Diagnosis}

LD can be very difficult to diagnose because the most common diagnostic tests are inaccurate approximately $50 \%$ of the time, especially sub-acutely or late in the disease process [43]. Without the characteristic EM to aid in diagnosing LD, blood tests, which are utilized to detect antibodies to LD, require increased accuracy, specificity, and sensitivity in preventing and treating LD [54]. LD requires a clinical diagnosis and health providers in areas with increased LD prevalence need to be aware of LD manifestations in people of all ethnicities and skin colors [43]. Healthcare providers assume based on recorded statistics that LD is rare in African Americans and people of color, which translates into lower incidence rates reported for people of color who may be presenting with less characteristic symptoms [36]. Misdiagnosis, or late diagnosis, adversely affects health outcomes of people of color as patients living with chronic LD have reported severe decline in health status [46].

\section{Recommendations}

\section{Implications}

The primary concern related to these facts is that $\mathrm{LD}$ is reported with lower frequency in populations in Appalachia with darker skin tones but may be due to discrepancies in diagnosis by healthcare professionals, among other factors [36]. In order for healthcare professionals to address the issue for their patients, we provide the following recommendations. We define the characteristic of darker skin tones as a "high VL score", or a score of $\geq 19$ on the von Luschan chromatic scale Figure 1, which has a maximum score of 38 for the darkest possible skin pigmentation expected in a patient [36, 56]. It is noted that while the von Luschan scale has been phased out of medical use for racial demographic categorization, it is an inexpensive and sufficient tool for skin tone as an independent characteristic [56]. One reason that the initial symptoms of LD are potentially overlooked in these patients is that the EM that normally characterizes the acute Ixodes tick bite associated with $B$. burgdorferi infection is less visible during general examination of high VL scoring patients [36-49].

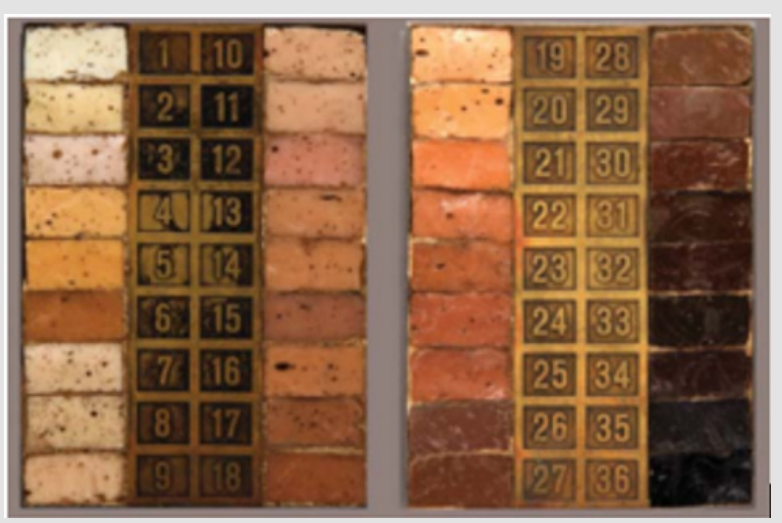

Figure 1: Image of the original tiles used for the von Luschan scale. The specific wavelengths of each pigment has been identified by spectrophotometry. 
Many individuals in Appalachia who self-identify as minorities according to the U.S. Census Bureau are projected to be high VL scoring patients based on the Bureau's average physical criteria [36-56]. Additionally, certain minorities, such as African American and Latinx individuals, have shown lower rates of receiving regular medical service due to lack of access and/or an inability to afford healthcare, as well as higher rates of occupations or lifestyles that carry greater risk of exposure to the vectors of LD [49]. The lack of physician access as well as the increased risk of exposure for dark-skinned patients is of interest to us as a potential cause of undiagnosed LD. This delay in diagnosis is a contributing factor in the increased prevalence of LD in patients who are high VL scorers, as well as the possibility of LD to be mistaken for other conditions due to the similarities in patient presentations $[57,58]$.

\section{Areas of Focus}

In order to confirm the need for further research into the prevalence of undiagnosed acute and late phase LD in dark-skinned patients, we recommend a systematic two-step research approach that is justified by our observations. Both steps allow for the anonymization of the patients involved and are expected to pose no health risk to the participants. The combined data collected from these studies would allow quantitative correlations to be made regarding skin tone and the prevalence of undiagnosed and chronic LD in the Appalachian region. The first step would focus on the results of a medical survey of patients known to have LD to see if there is a significantly greater prevalence of late phase LD in von Luschan scale scorers of $\geq 19$ out of 38 compared to von Luschan scale scorers of below 19 [36-57].

The second step would focus on willing undiagnosed participants who would be tested for acute and late phase LD, with an interest in the correlation between positive results and higher von Luschan scores [36-56]. The test for LD would involve use of the Lyme antigen test, which is specific for the Borrelia bacterial outer surface protein A (OpsA) and will show a positive result if the Borrelia spirochete is present in the patient's blood [5759]. This test has a higher sensitivity than the Enzyme-Linked Immunosorbent Assay (ELISA) and Western Blot methods, which are only specific for the host's antibody response to Borrelia and are less accurate [57]. Following a positive test result, a physician must then confirm LD through clinical investigation into the patient's other physical symptoms, environment, lifestyle, and relevant background $[57,58]$.

\section{Potential Consequences and Related Recommendations}

A positive correlation in both of the above research endeavors would justify the inclusion of LD testing and preventive medicine for LD for all patients in Appalachia who score $\geq 19$ on the von Luschan scale [36-57]. We advise physicians in the fields of primary care and emergency medicine in particular to note correlating factors between patients who have high VL scores and the prevalence of LD [36-49]. Due to the high overlap between patients who are dark- skinned and are minorities, the greatest chance of encountering these at-risk patients is during primary care or Emergency Department (ED) visits. The results of the recommended research would determine whether primary care and ED physicians in Appalachia should: incorporate testing for LD as part of general preventive medical treatment, prescribe preventive treatment for at-risk patients, check for signs of Ixodes tick during a general exam in at-risk patients, and prescribe antibiotics for patients that show signs of Ixodes bites [57,58]. Additionally, at-risk patients should be educated about the prevention of Lyme and self-monitoring for signs of an Ixodes tick bite or symptoms of LD, as well as lifestyle factors that increase risk of exposure to the vectors of LD and Borrelia [49-58].

\section{References}

1. (2019) The Centers for Disease Control and Prevention. Lyme Disease.

2. (2013) Lyme Disease Association. Lyme In 80+ Countries Worldwide.

3. Farlow J, Postic D, Smith KL, Jay Z, Baranton G, et al. (2002) Strain typing of borrelia burgdorferi, borrelia afzelii, and borrelia garinii by using multiple-locus variable-number tandem repeat analysis. J Clin Microbiol 40(12): 4612-4618.

4. Comstedt P, Jakobsson T, Bergström S (2011) Global ecology and epidemiology of Borrelia garinii spirochetes. Infe Ecol \& Epidemiol.

5. (2019) The Centers for Disease Control and Prevention. Borrelia mayonii.

6. (2019) Lyme Disease Association. About Lyme Disease Symptoms.

7. Lyme Disease Symptoms.

8. (2018) The Centers for Disease Control and Prevention. Signs and symptoms of untreated Lyme disease.

9. Feder HM, Johnson B, O'Connell S, Shapiro ED, Steere AC, et al. (2007) A critical appraisal of "chronic lyme disease". N Engl J Med 357: 14221430.

10. Lantos PM, Nigrovic LE, Auwaerter PG, Fowler VG, Ruffin F, et al. (2015) Geographic expansion of lyme disease in the southeastern united states, 2000-2014. Open Forum Infect Dis 2(4).

11. Johnson RC (1996) Medical Microbiology. In: S Baron (Eds.), ( $4^{\text {th }}$ edn.). Galveston (TX): University of Texas Medical Branch at Galveston.

12. Guerra M, Walker E, Jones C, Paskewitz S, Cortinas RM, et al. (2002) Predicting the risk of Lyme disease: Habitat suitability for Ixodes scapularis in the north central united states. Emerg Infect Dis 8(3): 289297.

13. (2019) The Centers for Disease Control and Prevention. Tickborne Diseases of the United States.

14. Adrion ER, Aucott J, Lemke KW, Weiner JP (2015) Health care costs, utilization and patterns of care following lyme disease. PLOS ONE 10(2).

15. Palmieri JR, King S, Case M, Santo A (2013) Lyme disease: Case report of persistent Lyme disease from Pulaski county, Virginia. Internati Med Case Rep J 6: 99-105.

16. Palmieri JR, Warehime J, King S, Doolan A, Reynolds D, et al. (2015) Undetected Lyme Disease (LD) and Southern Tick-Associated Rash Illness (STARI) in populations of southwest Virginia, leading to delayed treatment and an increased incidence of chronic infection. Austin J Microbiol 1(1): 1005.

17. Gilbert L (2010) Altitudinal patterns of tick and host abundance: A potential role for climate change in regulating tick-borne diseases? Oecologia 162: 217-225.

18. Brownstein JS, Holford TR, Fish D (2005) Effect of climate change on Lyme disease risk in North America. HHS Public Access 2(1): 38-46.

19. Appalachian Regional Commission. The Appalachian Region. 
20. Wilkey J (2017) What/Where/Who is Appalachia. This Appalachian Life

21. Shapiro ED (2008) Lyme disease. In: Finn A, Pollard AJ (Eds.), Hot topics in infection and immunity in children IV. Heidelberg: Springer pp: 185 195.

22. (2011) New York State Department of Health, Information for a healthier New York. Be Tick Free A- Guide for Preventing Lyme Disease.

23. Bope ET, Kellerman RD (2014) Conn's Current Therapy. $1^{\text {st }}$ (edn.). Elsevier.

24. (2014) Centers for Disease Control and Prevention. Tick Borne Diseases of the United States: A Reference Manual for Healthcare Providers. $\left(2^{\text {nd }}\right.$ edn.).

25. Shelus V (2012) Tick-borne disease risk along the Appalachian trail Retrieved from Retrieved from https://pdfs.semanticscholar.org/ c6e5/940ce0161d3b0363e9541989e5b98e609620.pdf.

26. Knoll JM, Ridgeway AC, Boogaerts CM, Burket III GA (2014) Appalachian trail Hikers' ability to recognize Lyme disease by visual stimulus photographs. Wilderness environmed 25(1): 24-28.

27. Johnson TL, Graham CB, Boegler KA, Cherry CC, Maes SE, et al. (2017) Prevalence and diversity of tick-borne pathogens in nymphal Ixodes scapularis (Acari: Ixodidae) in Eastern national parks. J Med Entomol 54(3): 742-751.

28. Eisen L, Wong D, Shelus V, Eisen RJ (2013) What is the risk for exposure to vector-borne pathogens in United States national parks? J Med Entomol 50(2): 221-230

29. Kilpatrick AM, Dobson AD M, Levi T, Salkeld DJ, Swei A, et al. (2017) Lyme disease ecology in a changing world: Consensus, uncertainty and critical gaps for improving control. Phil Trans R Soc Lond B Biol Sci 372(1722) pii 20160117.

30. Treesirichod A, Chansakulporn S, Wattanapan P (2014) Correlation between skin color evaluation by skin color scale chart and narrowband reflectance spectrophotometer. Ind J dermatol 59(4): 339-342.

31. Wanus S, Hemrungrote S, Chaisricharoen R (2015) Cloud service for detection of human skin color. In Communications and Information Technologies (ISCIT), $15^{\text {th }}$ International Symposium on, pp. 9-12. IEEE.

32. Virginia Department of Education. (n.d.) Frequently Asked Questions.

33. (2018) U.S. Census Bureau. Race and Ethnicity in Virginia.

34. Fix AD, Peña CA, Strickland GT (2000) Racial differences in reported Lyme disease incidence. Am J epidemiol 152(8): 756-759.

35. Killilea ME, Swei A, Lane RS, Briggs CJ, Ostfeld RS (2008) Spatial dynamics of Lyme disease: A review. EcoHealth 5(2): 167-195.

36. Christina F, Fix AD, Peña CA, Strickland GT (2002) Mapping LD incidence for diagnostic and preventive decisions, Maryland. Emerg infect dis 8(4): 427.

37. Coyle BS, Strickland GT, Liang YY, Peña C, McCarter R, et al. (1996) The public health impact of Lyme disease in Maryland. J Infect Dis 173(5): $1260-1262$.

38. (2018) Lyme disease.

ISSN: 2574-1241

DOI: 10.26717/BJSTR.2019.21.003583

James R Palmieri. Biomed J Sci \& Tech Res

This work is licensed under Creative

Commons Attribution 4.0 License

Submission Link: https://biomedres.us/submit-manuscript.php
39. Schwartz AM, Hinckley AF, Mead PS, Hook SA, Kugeler KJ (2017) Surveillance for Lyme disease-united states. MMWR Surveill Summ 66(22): 1-12.

40. Springer YP, Johnson PT J (2018) Large-scale health disparities associated with Lyme disease and human monocytic ehrlichiosis in the united states, 2007-2013. Plos one 13(9): e0204609.

41. (2014) U.S. Lyme disease reported cases 1990-2014. Lyme disease association.

42. Infectious Arthritis.

43. (2017) Lyme Disease: Symptoms, Treatment, and Prevention.

44. Li J, Kolivras KN, Hong Y, Duan Y, Seukep SE, et al. (2014) Spatial and temporal emergence pattern of Lyme disease in Virginia. Am J trop med hyg 91(6): 1166-1172.

45. Manni MF, Le Y, Morse W, Hollenhorstm SJ (2012) Shenandoah National Park visitor study: Summer and Fall 2011. Natural Resource Report NPS/NRSS/EQD/NRR-2012/584. National Park Service, Fort Collins, Colorado.

46. (2018) Outdoor Participation Report. Outdoor industry association.

47. Lyme Disease.

48. (2013) Virginia Department of Health. Exploring the Epidemiology, Clinical Aspects, and Prevention of Lyme Disease in Virginia.

49. Pachner AR (1989) Neurologic manifestations of lyme disease, the new "great imitator". Rev Infect Dis Suppl 6: S1482-S1486.

50. Minnesota lyme association. Symptoms, Diagnosis, \& Testing.

51. Nelson CA, Starr JA, Kugeler KJ, Mead PS (2016) Lyme disease in hispanics, united states, 2000-2013. Emerg infect dis 22(3): 522.

52. Liesbeth B, Goderis G, Vandevoorde J, Devroey D (2014) Relevance of chronic Lyme disease to family medicine as a complex multidimensional chronic disease construct: A systematic review. Inter J family med.

53. Wahlberg P, Granlund H, Nyman D, Panelius J, Seppälä L (1993) Late lyme borreliosis: Epidemiology, diagnosis and clinical features, Annals of Med 25(4): 349-352.

54. Swiatoniowski AK, Quillen EE, Shriver MD, Jablonski NG (2013) Technical Note: Comparing von Luschan skin color tiles and modern spectrophotometry for measuring human skin pigmentation. Am Journal Phy Anthropol 151(2): 325-330.

55. Treatment Issues and Guidelines. Lyme action network.

56. Cameron DJ, Johnson LB, Maloney EL (2014) Evidence assessments and guideline recommendations in lyme disease: The clinical management of known tick bites, Erythema Migrans rashes and persistent disease. Inter Lyme and Assoc Dis Soci.

57. Lyme antigen test.

58. Cobb RJ, Thomas CS, Pirtle WN, Darity WA (2016) Self-identified race, socially assigned skin tone, and adult physiological dysregulation: Assessing multiple dimensions of "race" in health disparities research. SSM Popul Health 2: 595-602.

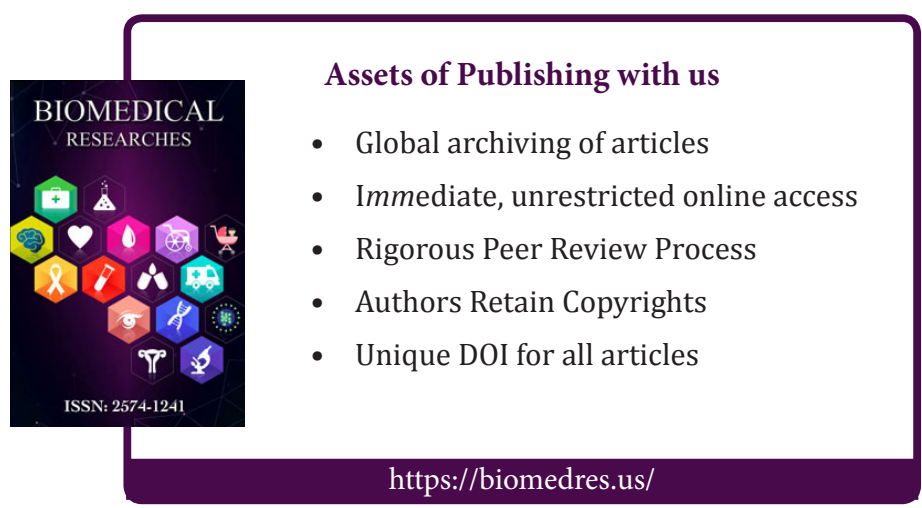

